Chapter 14

\title{
Ionic Liquids: "Green" Solvent for Catalytic Oxidations with Hydrogen Peroxide
}

\author{
Liangfang Zhu and Changwei Hu \\ Additional information is available at the end of the chapter \\ http://dx.doi.org/10.5772/51936
}

\section{Introduction}

Over the past decade, ionic liquids (ILs) have received great deal of attention as possible "green" replacement for volatile organic solvent mainly due to their nonmeasurable vapor pressure and good dissolubility for other salts. [1-5] Reaction types successfully performed in ILs include Diels-Alder, [6] Friedel-Crafts, [7] olefin hydrogenation, [8] hydroformylation, [9] , [10] oligomerization, [11] and Heck and Suzuki coupling reactions. [12] , [13] In addition to solvent, ILs may have multiple functions in catalytic reactions. They may act as catalyst, co-catalyst, support, or ligands for the catalytic process. [14] In particular, some "unexpected" effects have been observed in affecting the catalytic reaction pathway. For example, the cations/anions in ILs may be involved in the formation of the active species changing the reaction mechanism. [15] - [20] Understanding the functions of ILs in the catalytic reaction is of critical importance for deliberately modifying existed reaction system and exploiting new types of synthetic route by using this "green" solvent.

Catalytic oxidation is a class of commercially important reaction. As an environmentally benign oxidant, hydrogen peroxide $\left(\mathrm{H}_{2} \mathrm{O}_{2}\right)$ has been used for several catalytic oxidation. So far, significant improvements on the catalytic performance, in terms of yield and selectivity, have been observed using ILs as the solvent for the $\mathrm{H}_{2} \mathrm{O}_{2}$ oxidation reaction. [21] - [23] Actually, in many cases, ILs are active participant because the formation of radical species, stabilization of the charged reactive intermediate, and immobilization of the actual catalyst can be strongly affected by the presence of an ionic environment. In comparison with traditional organic solvent, the use of ILs in catalytic oxidation has been regarded as a new means for recycling the catalyst and enhancing the yield and selectivity of the product. Though a great number of catalytic oxidation have been performed in ILs, there are still rare examples which demonstrate how the ILs affect the reaction pathway and the reactivity. 
This chapter aims at summarizing the examples that concern the $\mathrm{H}_{2} \mathrm{O}_{2}$ oxidation reactions in ILs, in particular the benzene hydroxylation, alcohol oxidation, and olefin oxidation. The effects of ILs on the reaction pathway and the selectivity are discussed, drawing to the conclusion that ILs are offering unique properties as solvent by recycling the catalyst and enhancing the yield and selectivity of the product. What should be pointed out is that the examples are limited as far as possible to those that inform the readers' understanding of the role of ILs in the $\mathrm{H}_{2} \mathrm{O}_{2}$ oxidation reaction. We apologize that some fine work is not covered, and we hope to stimulate more discussions in the future.

\section{Benzene hydroxylation}

Direct hydroxylation of benzene to phenol with $\mathrm{H}_{2} \mathrm{O}_{2}$ has been extensively investigated owing to the reduced reaction steps and environmentally benign byproduct of water when comparing to the commercial cumene process for phenol production. One of the fundamental targets in this intriguing investigation is to enhance the utilization efficiency of $\mathrm{H}_{2} \mathrm{O}_{2}$ and the selectivity of phenol. The low efficiency of $\mathrm{H}_{2} \mathrm{O}_{2}$ always derives from the fast decomposition of $\mathrm{H}_{2} \mathrm{O}_{2}$, and the low product selectivity is mainly originated from the over-oxidation of phenol. Studies have shown that solvents used in the hydroxylation play an important role on enhancing both the $\mathrm{H}_{2} \mathrm{O}_{2}$ efficiency and the product selectivity. For example, water was the solvent in the traditional Fenton's reagent $\left(\mathrm{Fe}^{\mathrm{II}}-\mathrm{H}_{2} \mathrm{O}_{2}\right)$ catalyzed hydroxylation, [24] whereas, the decomposition of $\mathrm{H}_{2} \mathrm{O}_{2}$ was very fast. [25] The selectivity to phenol was rather poor in the aqueous solution since phenol is more reactive toward oxidation than benzene itself. Acetonitrile and acetic acid were then used as the solvents for most of the catalyzed hydroxylation of benzene, [26] [28] and a biphasic water-acetonitrile (1:1) system was developed to decrease the over-oxidation of phenol. [29] In Bianchi et al.'s work, [30] sulfolane was believed to form complexes with phenolic compounds inducing increased selectivity to phenol.

In addition to organic solvents, Peng et al. [31] introduced a biphasic aqueous-imidazoliumbased IL system for benzene hydroxylation in the presence of ferric tri(dodecanesulfonate) catalyst (Figure 1). In this aqueous- $\left[\mathrm{C}_{\mathrm{n}} \mathrm{mim}\right][\mathrm{X}]\left(\mathrm{n}=4,8,10 ; \mathrm{X}=\mathrm{PF}_{6}, \mathrm{BF}_{4}\right)$ IL biphasic system, both the catalyst and benzene were dissolved in the IL, whereas, $\mathrm{H}_{2} \mathrm{O}_{2}$ was mainly dissolved in aqueous phase. The produced phenol was extracted into water phase, minimizing the over-oxidation. A highest yield of $54 \%$ and selectivity of $100 \%$ to phenol was obtained in the aqueous-IL biphasic system. However, as $\mathrm{H}_{2} \mathrm{O}_{2}$ was existed in the IL-free aqueous phase, the active oxidizing agent were deemed to be radical species. Therefore, this biphasic system was not operative for the hydroxylation of toluene: only $1 \%$ of toluene was converted to benzaldehyde although its selectivity reached $100 \%$.

In our work, [25] , [32] a benzene-triethylammonium acetate ([ $\left.\left.\mathrm{Et}_{3} \mathrm{NH}\right]\left[\mathrm{CH}_{3} \mathrm{COO}\right]\right)$ IL biphasic system was constructed for the benzene hydroxylation (Figure 2). The Fenton-like reagent $\left(\mathrm{Fe}^{\mathrm{III}}-\mathrm{H}_{2} \mathrm{O}_{2}\right)$ existed in the IL phase and most of the phenol was extracted to the benzene layer. The $\left[\mathrm{Et}_{3} \mathrm{NH}\right]\left[\mathrm{CH}_{3} \mathrm{COO}\right] \mathrm{IL}$ was found to be stable in the water- and oxygenrich environment. Benzene acted as both the substrate and the extractant in the hydroxyla- 
tion reaction. In comparison with the aqueous-IL biphasic system, the continuous extraction of phenol by benzene from $\left[\mathrm{Et}_{3} \mathrm{NH}\right]\left[\mathrm{CH}_{3} \mathrm{COO}\right] \mathrm{IL}$ protected phenol from further oxidation by directly avoiding the contact of phenol with the catalyst and oxidant. As a result, moderate yield (20\%, based on benzene converted, excluding evaporated) and high selectivity (> $99.5 \%$ ) of phenol were obtained in the IL-benzene biphasic system.

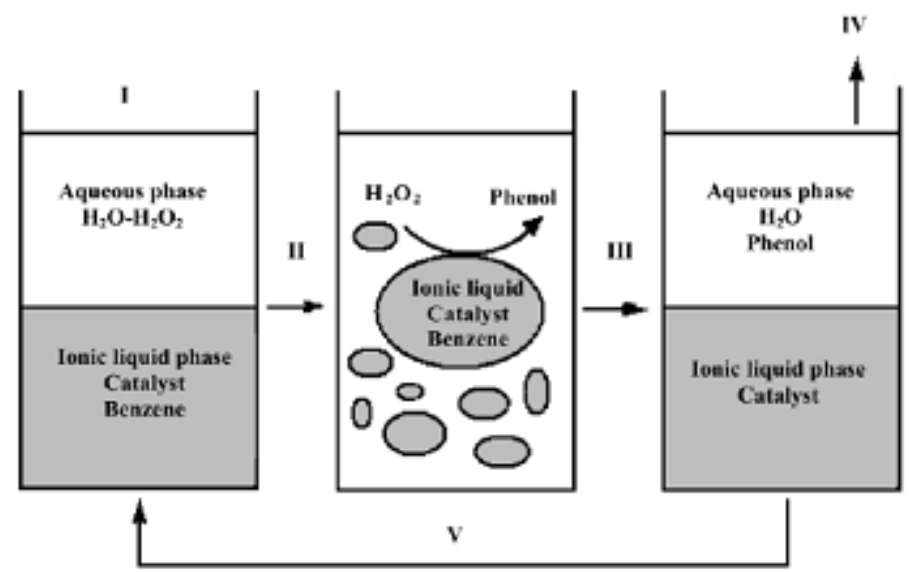

Figure 1. A schematic representation of the aqueous-IL biphasiccatalytic reaction system for benzene hydroxylation to phenol with $\mathrm{H}_{2} \mathrm{O}_{2}$. Step I charging; Step II reaction; Step III still; Step IV recovery of phenolvia extraction; Step V the IL and catalyst are reused for anotherreaction cycle. IL $=\left[C_{n} \operatorname{mim}\right][X]\left(n=4,8,10 ; X=\mathrm{PF}_{6}, \mathrm{BF}_{4}\right)$. [31] Reprinted with permission from ref. 31. Copyright (C2003, Royal Society of Chemistry.

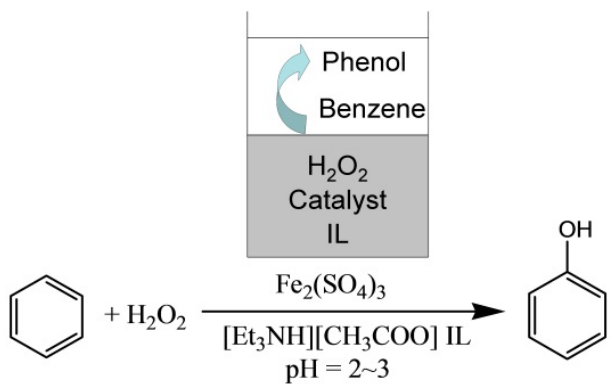

Figure 2. Schematic illustration of the benzene- $\left[\mathrm{Et}_{3} \mathrm{NH}\right]\left[\mathrm{CH}_{3} \mathrm{COO}\right] \mathrm{IL}$ biphasic system for benzene hydroxylation to phenol with $\mathrm{H}_{2} \mathrm{O}_{2}$. [25]

Moreover, the $\left[\mathrm{Et}_{3} \mathrm{NH}\right]\left[\mathrm{CH}_{3} \mathrm{COO}\right] \mathrm{IL}$ exhibited retardation performance for the decomposition of $\mathrm{H}_{2} \mathrm{O}_{2}$ and protection performance for the over-oxidation of phenol. From a molecular aspect, the $\mathrm{CH}_{3} \mathrm{COO}$ - anions of $\left[\mathrm{Et}_{3} \mathrm{NH}\right]\left[\mathrm{CH}_{3} \mathrm{COO}\right] \mathrm{IL}$ were found to be coordinated with the Fe ions, forming Fe complexes, by virtue of the solution of Fenton-like reagent in the IL 
phase (Figure 2). [25] Such coordination "anchored" the catalyst in the IL, affecting the subsequent activation of benzene and $\mathrm{H}_{2} \mathrm{O}_{2}$. A higher electrophilicity of the Fe-complexes was favorable for the interaction of $\mathrm{H}_{2} \mathrm{O}_{2}$ with the Fe center, which may be the origin of the retardation role of IL on the decomposition of $\mathrm{H}_{2} \mathrm{O}_{2}$. High-valent $\mathrm{Fe}^{\mathrm{IV}}$-oxo species, formed from the $\mathrm{O}-\mathrm{O}$ bond homolysis of a $\mathrm{Fe}^{\mathrm{III}}-\mathrm{OOH}$ intermediate, was found to be the main active oxidizing species in the ionic environment rather than the widely accepted oxidizing species of hydroxyl radical $\left(\mathrm{OH}^{*}\right)$ in an aqueous Fenton system. The mechanism for hydroxylation of benzene in the $\left[\mathrm{Et}_{3} \mathrm{NH}\right]\left[\mathrm{CH}_{3} \mathrm{COO}\right] \mathrm{IL}$ was thus different from that occurred in aqueous solution (Figure 3) [32] : the activation of benzene was mainly achieved via the electrophilic attack by the $\mathrm{Fe}^{\mathrm{IV}}$-oxo species, rather than via hydrogen abstraction to form phenyl radical. Over-oxidation of phenol through $\mathrm{H}$-abstraction from $\mathrm{O}-\mathrm{H}$ of phenol by the $\mathrm{Fe}^{\mathrm{IV}}$-oxo species was partly prohibited by the hydrogen-bond interaction between phenol and the $\mathrm{CH}_{3} \mathrm{COO}$ anion. The electrophilic character of the $\mathrm{Fe}^{\mathrm{IV}}$-oxo species made the $\left[\mathrm{Et}_{3} \mathrm{NH}\right]\left[\mathrm{CH}_{3} \mathrm{COO}\right] \mathrm{IL}$ suitable for hydroxylation of other alkyl-benzenes. As an instance, the oxidation of toluene in $\left[\mathrm{Et}_{3} \mathrm{NH}\right]\left[\mathrm{CH}_{3} \mathrm{COO}\right] \mathrm{IL}$ resulted in the selective activation of benzene ring with the selectivity to methylphenols of about $62 \%$. [33]

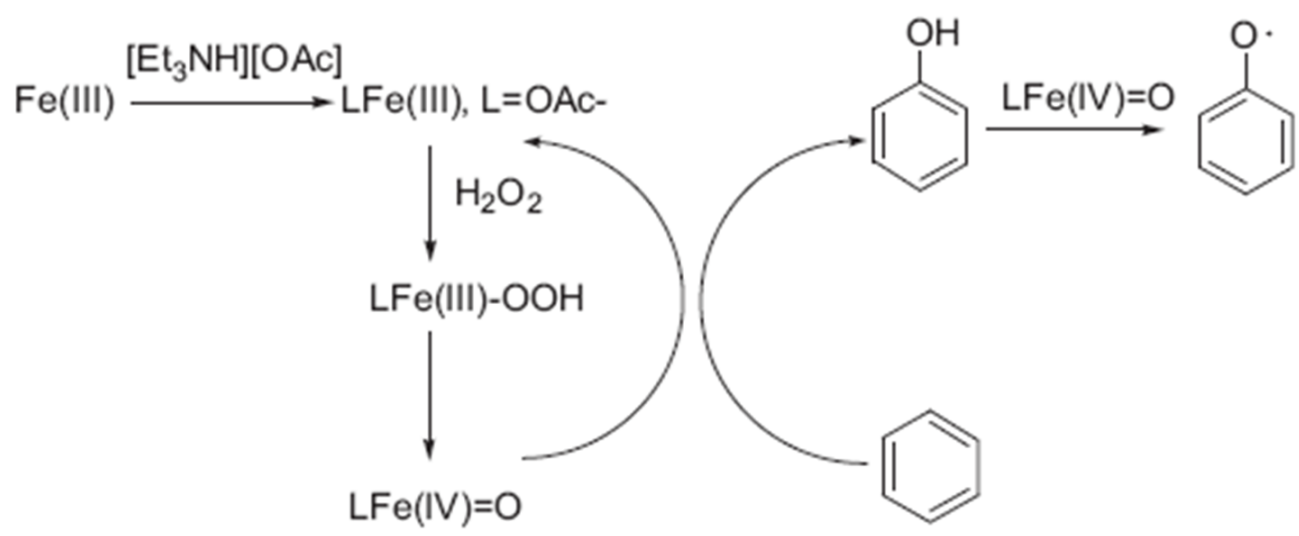

Figure 3. Mechanism for hydroxylation of benzene with $\mathrm{H}_{2} \mathrm{O}_{2}$ in $\left[\mathrm{Et}_{3} \mathrm{NH}\right.$ ] [OAc] system. [32] Reprinted with permission from ref. 32. Copyright (c) 2011, Elsevier Science Ltd.

In the benzene hydroxylation, both hydrophobic and hydrophilic ILs have shown the feasibility of acting as solvent for enhancing both the yield and selectivity of phenol when compared with that in aqueous solution (vide supra). The nature of the active oxidizing species is mostly depended on the dispersion of both $\mathrm{H}_{2} \mathrm{O}_{2}$ and the catalyst in the ILs because the generation of active species is thus influenced by the ionic environment. Furthermore, the combination of ILs with a second solvent (co-solvent), either traditional organic solvent or water, may offer opportunity for breaking the thermodynamic equilibrium by mass-transfer of the product from phase to phase. Most importantly, the ILs-solvent biphasic system provides opportunity for the stabilization of charged reactive intermediate and the protection of unstable product from over-oxidation. Therefore, the ILs-co-solvent biphasic system can be 
tentatively developed as solvent for a wide range of $\mathrm{H}_{2} \mathrm{O}_{2}$ oxidation reaction to meet specific requirements, for example, yield, selectivity, or solubility, etc.

\section{Alcohol oxidation}

The partial oxidation of alcohols to aldehydes, or secondary alcohols to corresponding ketones is a fundamental synthetic transformation in organic chemistry and is industrially important. [34] - [36] However, this transformation always suffers from drawbacks such as poor conversion and selectivity due to over-oxidation. Stable-free nitroxyl radicals such as $\operatorname{TEMPO}(2,2,6,6$-tetramethylpiperidine-1-oxyl) has recently emerged as a catalyst or co-catalyst to promote the formation of the catalytically active species for selective oxidation of alcohols to aldehydes or ketones where volatile organic solvents such as $\mathrm{CH}_{2} \mathrm{Cl}_{2}$ are frequently used. [37] - [44] However, the recycling of the quite expensive TEMPO is problematic due to the homogeneous character of the classic organic media. Replacement of organic solvents with ILs or immobilization of TEMPO on ILs provide alternative strategies for solving the above-mentioned problems. On the one hand, TEMPO can be anchored on ILs allowing the recycling of the catalyst; on the other hand, ILs provide advantages for increasing the selectivity of the product by promoting oxidation of alcohols to aldehydes but suppressing overoxidation of these aldehydes to acids.

In Wang et al.'s work, [45] oxidation of alcohols with $\mathrm{H}_{2} \mathrm{O}_{2}$ was performed in pyridiniumtetrafluoroborate([Bpy] $\mathrm{BF}_{4}$ ) IL. The catalyst (vanadate) and co-catalyst (TEMPO and sulfonic acid) were both grafted on [Bpy] $\mathrm{BF}_{4}$ IL. The functionalized IL showed good dissolubility in the [Bpy] $\mathrm{BF}_{4}$ solvent. The as-formed homogeneous mixture of $\mathrm{N}-\mathrm{n}$-dodecyl pyridinium vanadate, N-(propyl-1-sulfonic acid) pyridiniumtetrafluoroborate, and 4-(propanoate-TEMPO) pyridiniumtetrafluoroborate exhibited good activity and recyclability for alcohols oxidation.Jiang et al. [46] used the acetamido-modified TEMPO as catalyst for the selective oxidation of benzylic alcohols to aldehydes in the 1-n-butyl-3-methylimidazolium hexafluorophosphate ([bmim] [ $\left.\mathrm{PF}_{6}\right]$ ) IL (Figure 4). The [bmim] [ $\left.\mathrm{PF}_{6}\right]$ ILwas immiscible with $\mathrm{H}_{2} \mathrm{O}_{2}$, favoring the partial oxidation of alcohols to aldehydes but inhibiting the over-oxidation of aldehydes to acids by reducing the contact of the product with the oxidant. The miscibility of acetamido-TEMPO in [bmim] [ $\left.\mathrm{PF}_{6}\right]$ IL ensured good catalytic activity and efficient recycling of the catalyst. In comparison to the common organic solvents (ethyl acetate or chloroform), the yield of aldehydes was enhanced by about three times.

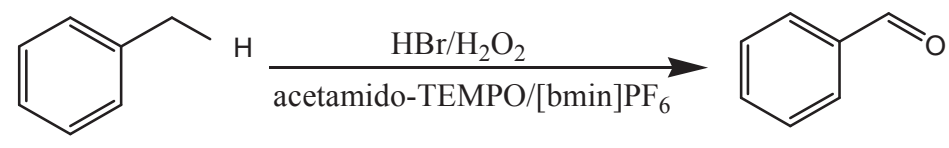

Selectivity $=99 \%$

Figure 4. Highly selective oxidation of benzyl alcohol to benzaldehyde with acetamido- $\mathrm{TEMPO} / \mathrm{HBr} / / \mathrm{H}_{2} \mathrm{O}_{2}$ in $[\mathrm{bmim}]$ $\left[\mathrm{PF}_{6}\right]$ IL. [46] 
The strategy of anchoring TEMPO on ILs has also been applied in other catalytic oxidation reaction. In Fall et al.'s work, [39] TEMPO was supported on ILs through click chemistry reaction (Figure 5a). The IL-supported catalyst exhibited good solubility in [HMIM] [BF $\left.{ }_{4}\right]$ IL and high activity for alcohol oxidation using bis(acetoxy)iodobenzene (BAIB) as the terminal oxidant. The catalyst can be recycled together with the IL without loss of the efficiency for several cycles. Karimi et al. [47] grafted TEMPO on SBA-15 solid support and then synthesized IL@SBA-15-TEMPO catalyst by physically confining 1-methyl-3-butylimidazolium ([Bmim] Br) IL within the mesopores of the TEMPO-modified SBA-15 (Figure 5b). The catalyst showed high activity, improved selectivity, and good recyclability for the oxidation of alcohols to aldehydesand ketones with $t$-butylnitrite (TBN) as oxidant in $\mathrm{AcOH}$. Although the catalytic performance of the TEMPO-ILs and ILs@support-TEMPO catalysts in the $\mathrm{H}_{2} \mathrm{O}_{2}$ oxidation reaction is not investigated, we may expect the strategy of fixing TEMPO onto ILs or solid support applicable in recycling the expensive catalyst in a wide range of catalytic reactions.

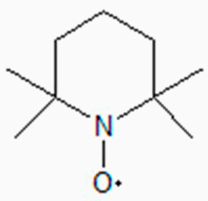

TEMPO

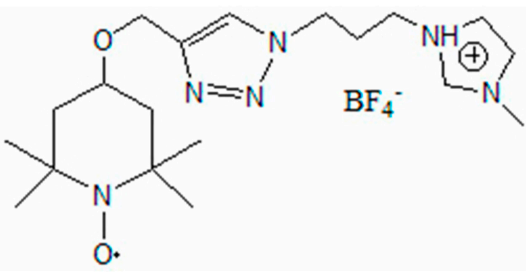

(a)

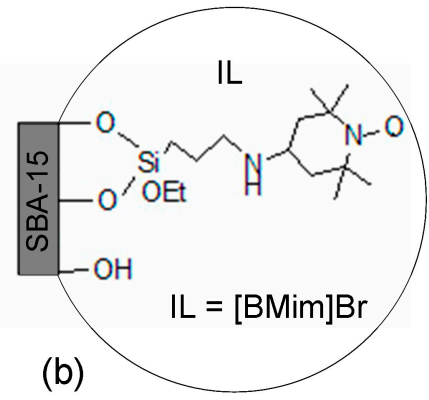

(b)

Figure 5. Strategies for immobilizing TEMPO on ILs. (a) IL supported TEMPO, [39] and (b) IL@SBA-15-TEMPO. [47]

In addition to TEMPO, the combination of various kinds of catalysts (i.e., inorganic salts, transition metal complexes, and oxides) with ILs has shown prospect as effective catalytic system for the $\mathrm{H}_{2} \mathrm{O}_{2}$ oxidation reaction. Chhikara et al. [48] synthesized imidazolium-based phosphotungstate catalyst (Figure 6,1 ) by grafting phosphotungstate onto imidazolium-based IL, which showed good catalytic performance in the homogeneous oxidation of secondary alcohols in the 1-butyl-3-methylimidazolium tetrafluoroborate ([bmim] [BF 4$]$ ) IL (Figure 6a). All of the secondary alcohols were converted to corresponding ketones in good to excellent yields. Oxidation of primary alcohol, i.e. benzyl alcohol, produced benzaldehydein good yield (78\%), or benzoic acid in high yield (96\%) after increasing the $\mathrm{H}_{2} \mathrm{O}_{2}$ amount (Figure 6b). The IL and catalyst after the extraction of the products could be reused for further catalytic oxidation.

Bianchini et al. [49] described the oxidation of some secondary alcohols to their ketones with $\mathrm{H}_{2} \mathrm{O}_{2}$ catalyzed by methyltrioxorhenium(VII)(MTO) or polymer supported-MTO in [bmim] $\left[\mathrm{PF}_{6}\right]$ or 1-ethyl-3-methylimidazolium bis-triflic amide ([emim] $\left.\left[\mathrm{Tf}_{2} \mathrm{~N}\right]\right)$ ILs. The supported catalyst was dispersed in the IL layer, which allowed the recycling of the catalyst and solvent system that could not be realized in a wide range of organic solvent. Moreover, in com- 
parison with ethanol or acetic acid, the activity of the catalyst in the ILs was obviously improved.

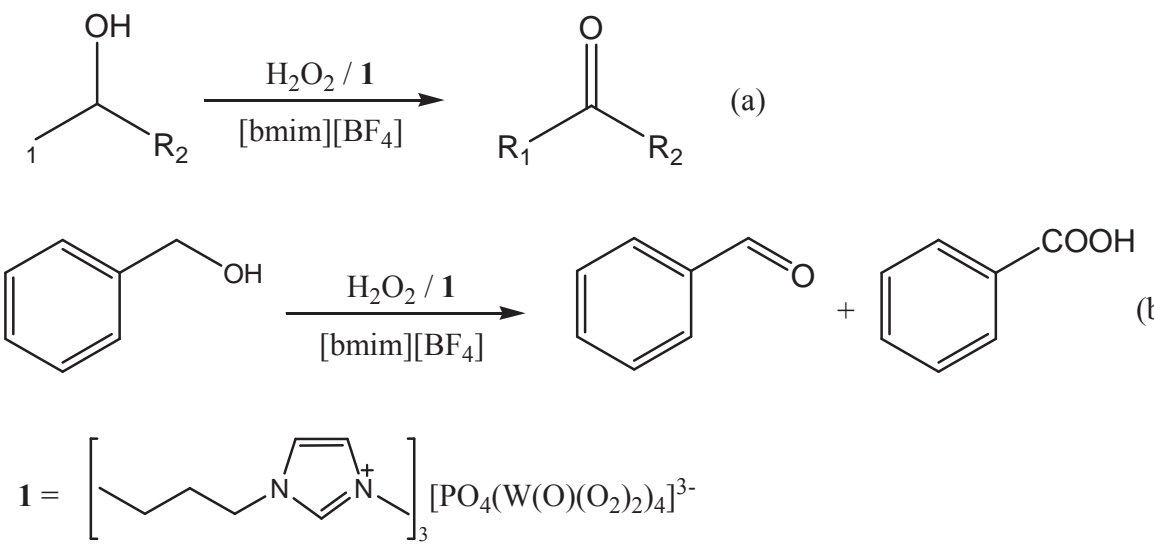

Figure 6. Oxidation of (a) secondary alcohols to ketones, and (b) benzyl alcohol to benzaldehyde and benzoic acid with imidazolium-based phosphotungstate (1) and $\mathrm{H}_{2} \mathrm{O}_{2}$ in [bmim] [BF $]$ IL. [48]

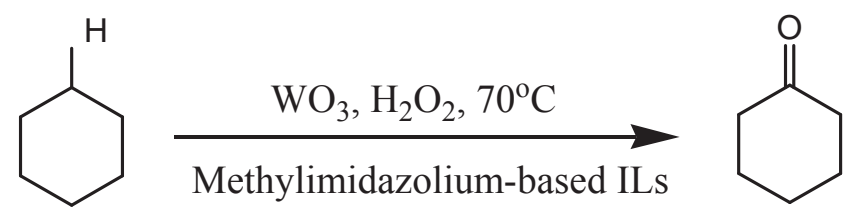

Figure 7. Selective oxidation of cyclohexanol to cyclohexanone with $\mathrm{H}_{2} \mathrm{O}_{2}$ in hydrophobic methylimidazolium-based ILs. [50]

Chen et al. [50] applied several hydrophobic methylimidazolium-based ILs in the oxidation of cyclohexanol to cyclohexanone with $\mathrm{H}_{2} \mathrm{O}_{2}$ using $\mathrm{WO}_{3}$ as the catalyst (Figure 7). The oxidation of cyclohexanol in the absence of ILs produced cyclohexanone with a moderate yield (42\%) or adipic acid at high cyclohexanol conversion. [51] In the biphasic cyclohexanol-ILs system, however, the ILs were found to effectively intensify cyclohexanol oxidation and resulted in $100 \%$ conversion of cyclohexanol with $100 \%$ selectivity to cyclohexanone. The high production of cyclohexanone can be explained by the fact that the oxidation of cyclohexanol occurred in aqueous phase contained $\mathrm{H}_{2} \mathrm{O}_{2}$ and $\mathrm{WO}_{3}$, whereas, the produced cyclohexanone was abstracted into the organic phase, minimizing the further oxidation of the product. Among the three kinds of methylimidazolium-based ILs (1-hydroxyethyl-3-methylimidazoliumchloride ([HOemim] $\mathrm{Cl}$ ); 1-hexyl-3-methylimidazoliumchloride ([Hmim] $\mathrm{Cl})$; and 1-octyl-3-methylimidazolium chloride ([Omim] $\mathrm{Cl}$,)) investigated, the [Omim] $\mathrm{Cl}$ IL exhibited the best solvent performance for enhancing the conversion of cyclohexanol when comparing with the traditional organic solvents (methanol, n-propanol, or acetone). Furthermore, a 
higher concentration of the [Omim] CIIL favored the transformation of cyclohexanol, giving evidence that the IL may be involved in the stabilization of the reaction intermediates in the catalytic process. Detailed investigation revealed that a longer alkyl chain of the IL increased the interaction between the solvent and the hydrophobic substrate, and a larger polarity of the IL increased the strength of coulombic forces arising in the solvation process. Both of these two factors improved the conversion of cyclohexanol considerably.

In this part, the anchoring of the actual catalyst on ILs offers opportunity for immobilizing catalyst with the solvent, allowing the recycling of the catalyst, especially some expensive reagent. This "anchoring" can be either chemical coupling or physical confinement. Chemical coupling requires special tailoring or functionalization of the ILs. The functionalized ILs show prospect as both catalyst and solvent for the $\mathrm{H}_{2} \mathrm{O}_{2}$ oxidation reaction. The physical confinement of ILs within some solid porous materials has dual effects on the catalytic oxidation: on the one hand, the ILs supply special microenvironment on affecting the reaction pathway; on the other hand, the micropores of the porous material allow the controlling of the selectivity of the product. In addition, we may expect structural modification of the functionalized ILs by deliberately varying the cations/anions to meet the distribution requirement of the substrate or product, which will be of great importance for increasing the yield and selectivity of the product. Beyond that, more synthetic method should be developed to support the actual catalyst in order to shed light on the effective utilization of the expensive reagent in future catalytic oxidation.

\section{Olefin oxidation}

Recently, significant improvements on the catalytic performance in some transition metalcatalyzed reactions have been observed using ILs as the solvent. [2] , [19] , [20] The roomtemperature ILs have emerged as environmentally benign reaction media as well as new vehicles for the immobilization of transition metal-based catalysts. Singh et al. [52] reported the $\mathrm{H}_{2} \mathrm{O}_{2}$ epoxidation of substrates containing both electron rich and deficient olefins catalyzed with meso-tetraarylporphyrin iron(III) chlorides ([TAPFe(III)Cl]) in imidazolium ILs. The active oxidizing species depended on the substrate used (Figure 8): the ferric peroxy anions (TAP-Fe ${ }^{\mathrm{III}}-\mathrm{OO}^{-}$) were effective intermediates in the epoxidation of electron deficient olefins, whereas the high valent oxoferrylporphyrin $\pi$-cation radicals $\left(\mathrm{TAP}-\mathrm{Fe}^{\mathrm{IV}}=\mathrm{O}^{\bullet+}\right.$ ) were involved in the epoxidation of electron rich olefins. The ILs provide special microenvironment via the interactions between their cations and anions, where the active intermediates could be fast generated from TAPFe ${ }^{\mathrm{III}} \mathrm{Cl} /[\mathrm{Bmim}]\left[\mathrm{PF}_{6}\right]$ and $\mathrm{H}_{2} \mathrm{O}_{2}$ and well stabilized in the $[\mathrm{Bmim}]\left[\mathrm{PF}_{6}\right] \mathrm{IL}$. The nature of the anions in the ILs played an important role on the activity of the catalyst.

Han and coworkers [53] synthesized novel $\mathrm{Ni}^{2+}$-containing 1-methyl-3- [(triethoxysilyl)propyl] imidazolium chloride (TMICl) IL immobilized on silica to catalytic oxidation of styrene to benzaldehyde with $\mathrm{H}_{2} \mathrm{O}_{2}$ under solvent-free condition (Figure 9). With the aid of the IL, both hydrophobic reactant and the hydrophilic reactant were accessible to the active sites of 
the catalyst: styrene and $\mathrm{H}_{2} \mathrm{O}_{2}$ are miscible with the $\mathrm{IL}$, and the $\mathrm{Ni}^{2+}$ was coordinated by the immobilized IL that allowed both reactants to access to active sites of the catalyst effectively. Under solvent-free condition, the conversion of styrene reached $18.5 \%$ and the selectivity to benzaldehyde was as high as $95.9 \%$ on the IMM-TMICl-Ni ${ }^{2+}$ catalyst.

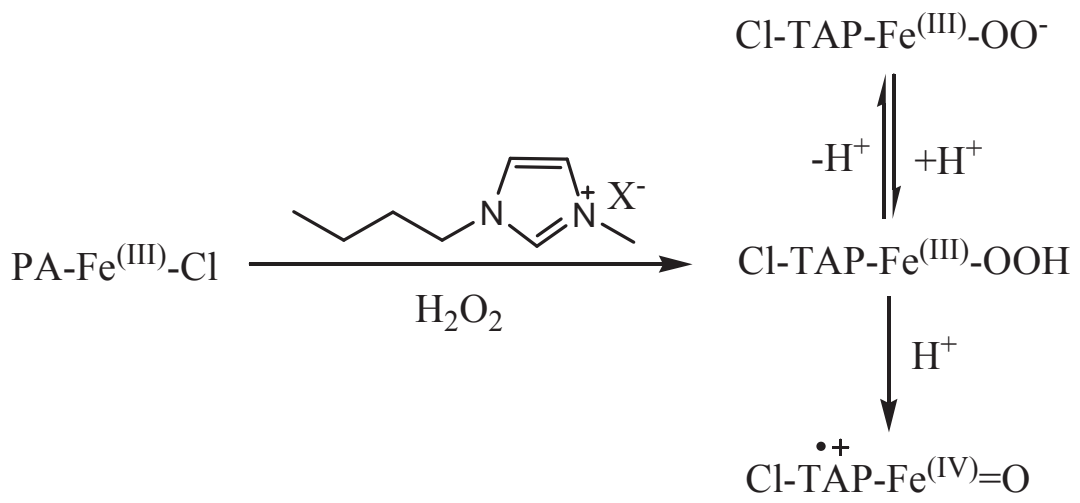

Figure 8. Mechanism for the generation of the reactive intermediate from TAPFe"' $\mathrm{Cl} /[\mathrm{Bmim}]\left[\mathrm{PF}_{6}\right]$ and $\mathrm{H}_{2} \mathrm{O}_{2}$. [52]

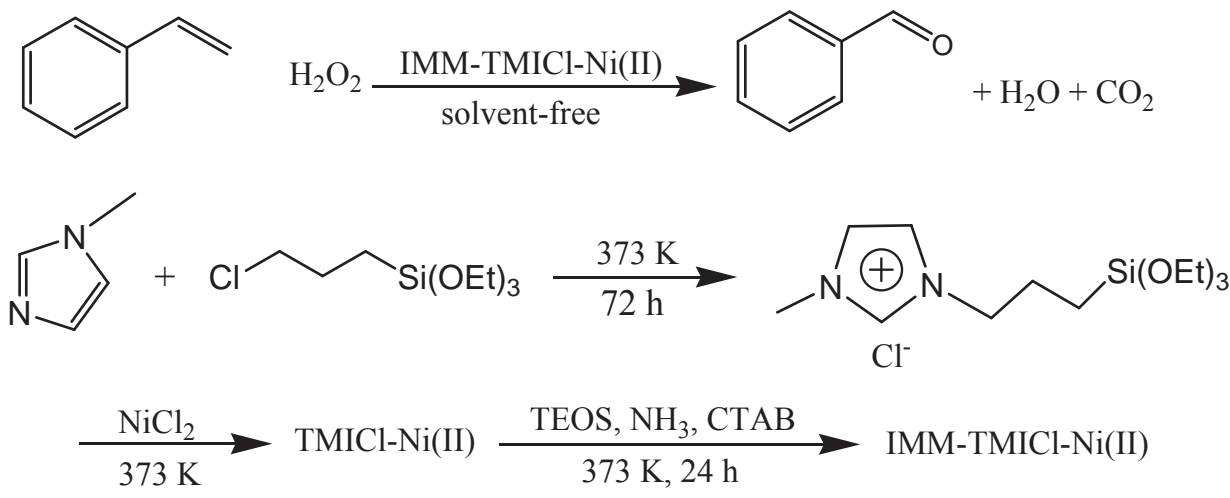

Figure 9. Schematic illustrations of (a) oxidation of styrene to benzaldehyde with $\mathrm{H}_{2} \mathrm{O}_{2}$ under solvent-free condition, (b) synthesis of IMM-TMICl-Ni(II) catalyst by grafting $\mathrm{Ni}^{2+}$ on IL-Silica. [53]

Some more examples are given by combining ILs with metal peroxides or polyoxometalates in the catalytic epoxidation of olefins with $\mathrm{H}_{2} \mathrm{O}_{2}$. In Yamaguchi et al.'s work, [54] peroxotungstate was immobilized on dihydroimidazolium-based IL-modified $\mathrm{SiO}_{2}$ (Figure 10). The as-prepared catalyst showed high activity and selectivity for epoxidation of a wide range of olefins. Radical mechanism was excluded for the IL-involved epoxidation in $\mathrm{CH}_{3} \mathrm{CN}$ solvent. Berardi et al. [55] embedded the catalytically active $\left[\gamma-\mathrm{SiW}_{10} \mathrm{O}_{36}(\mathrm{PhPO})_{2}\right]{ }^{4-}$ polyanions in 
the hydrophobic IL ([bmim] [[PF $]$ or hydrophilic IL [bmim] $\left.\left[\left(\mathrm{CF}_{3} \mathrm{SO}_{2}\right)_{2} \mathrm{~N}\right]\right)$. The catalyst gave out high yield and selectivity for epoxidation of olefins under microwave irradiation in the hydrophilic IL (Figure 11). The catalyst can be recycled with the catalytic IL phase. Liu et al. [56] demonstrated the role of [bmim] $\left[\mathrm{PF}_{6}\right] \mathrm{IL}$ as an activator for efficient olefin epoxidation with $\mathrm{H}_{2} \mathrm{O}_{2}$ catalyzed by Keggin polyoxometalate [bmim] ${ }_{3} \mathrm{PW}_{12} \mathrm{O}_{40}$ (Figure 12). In the IL, the interaction between the anions and the cations supplied a special microenvironment, accelerating the generation of the active peroxotungstate $\left[\mathrm{PO}_{4}\left\{\mathrm{~W}(\mathrm{O})(\mathrm{O})_{2}\right\}_{4}\right]^{3-}$ species from $[\mathrm{bmim}]$ ${ }_{3} \mathrm{PW}_{12} \mathrm{O}_{40}$ and $\mathrm{H}_{2} \mathrm{O}_{2}$. In some sense, the [bmim] [ $\left.\mathrm{PF}_{6}\right]$ IL could be considered as a co-catalyst to promote the formation of active species for epoxidation. Both of the TOF and selectivity for olefin epoxidation were significantly enhanced in the IL compared to that of traditional organic solvents, e.g., 289 times TOF and 1.3 times selectivity as found in $\mathrm{CH}_{2} \mathrm{Cl}_{2}$ for the epoxidation of cis-cyclooctene. The utilization efficiency of $\mathrm{H}_{2} \mathrm{O}_{2}$ reached as high as $87 \%$.

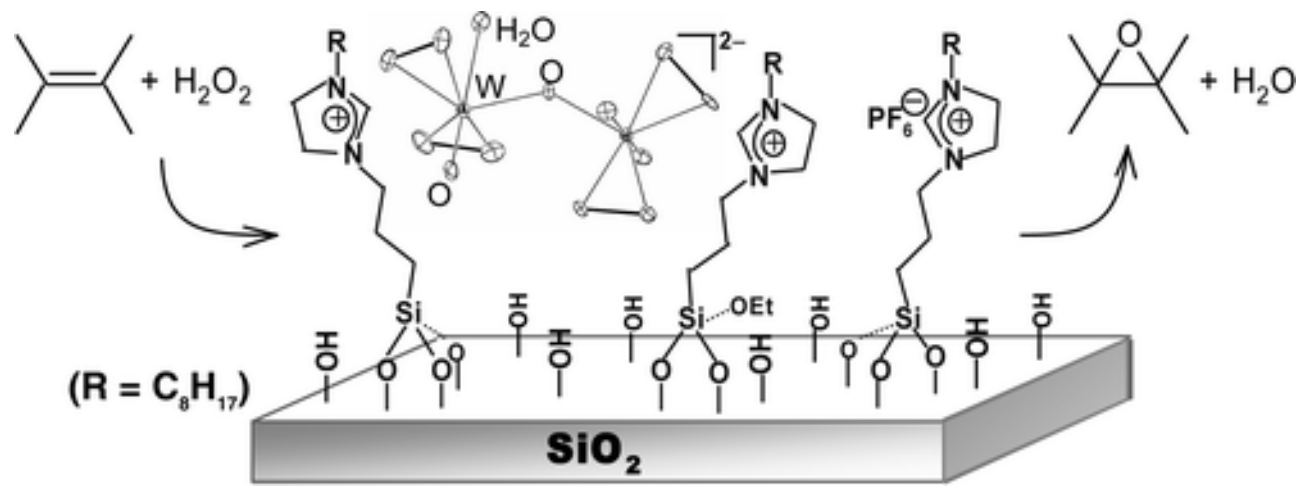

Figure 10. Epoxidation of Olefins with $\mathrm{H}_{2} \mathrm{O} 2$ catalyzed by peroxotungstate immobilized on IL-modified $\mathrm{SiO}_{2}$. [54] Reprinted with permission from ref.54. Copyright (c) 2005,AmericanChemical Society.

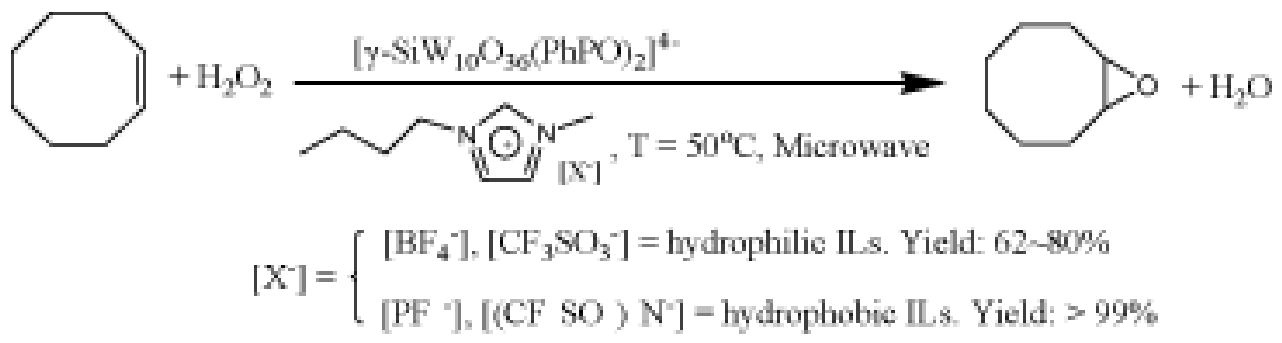

Figure 11. Epoxidaton of cis-Cyclooctene with $\mathrm{H}_{2} \mathrm{O}_{2}$ and polyoxometalates in both hydrophilic and hydrophobic ILs. [55]

Numerous examples have shown that ILs are offering unique properties in the transition metal-, metal peroxide- or polyoxometalates-catalyzed oxidation of olefins with $\mathrm{H}_{2} \mathrm{O}_{2}$. The 
ILs supply special environment for the generation and stabilization of the active intermediate, or act as support for immobilizing and recycling the actual catalyst, both of which are necessary for performing effective catalytic oxidation. By delicately designing the combination of catalyst, support and ILs, the interactions between the hydrophobic substrate, hydrophilic oxidant, and the active site could be reinforced, intensifying the catalytic efficiency of the oxidation reaction.

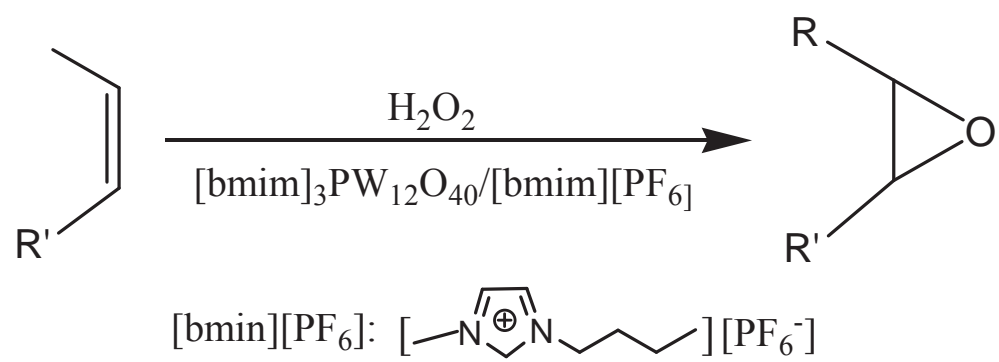

Figure 12. Epoxidation of olefins with $\mathrm{H}_{2} \mathrm{O}_{2}$ and polyoxometalates in [bmim] [PF $]$ IL. [56]

\section{Conclusion}

Catalytic oxidations have been widely studied in ionic liquids, and much of this interest is centered on the possible use as "green" alternatives to traditionally used volatile organic solvents. This chapter summarizes limited examples that illustrate the applications of ILs in the catalytic oxidation using $\mathrm{H}_{2} \mathrm{O}_{2}$ as the oxidant, in particular benzene hydroxylation, alcohol oxidation, and olefin oxidation. We focus our discussion on understanding how the unusual solvent environment provide solute species that affect the reactions occurred in them.

As innocent and non-vaporized solvents, ILs provide good solubility to salts and most of the hydrophobic substrate, endowing them good solvent for the transition metal complexes-, peroxides-, oxides-, polyoxometalates-, or organic molecules-catalyzed oxidation. The miscibility of ILs with water and organic molecules can be elaborately tuned by varying the cations/anions (i.e., length of alkyl chain, polarity, etc.). The interactions between ILs and the substrate, catalyst, oxidant, even reaction intermediate, make the ILs act as multi-functional solvent for the $\mathrm{H}_{2} \mathrm{O}_{2}$ involved catalytic oxidation. The cations/anions in ILs may influence the reaction pathway by stabilization of the charged transition state, active species or ligands which are necessary for many oxidation reaction. The structure of ILs can also be specially tailored to support actual catalyst and/or co-catalyst for effective recycling. The functionalized ILs have prospect as both catalyst and solvent for the $\mathrm{H}_{2} \mathrm{O}_{2}$ oxidation reaction. The grafting of ILs on some solid porous materials may improve the microenvironment of the reactive site, affecting the reaction pathway by increasing the selectivity of the product. Most importantly, the combination of ILs and a co-solvent (water or organic solvent) al- 
lows the mass transfer of unstable product from oxidative environment, protecting the product from over-oxidation by $\mathrm{H}_{2} \mathrm{O}_{2}$. As a conclusion, the integration of the multiple benefits from ILs will provide a greener scenario for recycling the catalyst and solvent, as well as improving the yield and selectivity of the product. We may expect novel synthetic strategy for functionalized ILs and their elaborate combination with prevailing catalytic materials for applications in a wide range of catalytic oxidation in future.

\section{Acknowledgments}

The financial support from the National Natural Science Foundationof China (No. 20901053 and 20872102) and PCSIRT (No. IRT0846) are greatly appreciated.

\section{Author details}

Liangfang Zhu and Changwei $\mathrm{Hu}^{*}$

*Address all correspondence to: changweihu@scu.edu.cn or chwehu@mail.sc.cninfo.net

Key Laboratory of Green Chemistry and Technology, Ministry of Education, College of Chemistry, Sichuan University, Chengdu, P.R. China

\section{References}

[1] Anastas, P. T.; Warner, J. C., Green Chemistry: Theory and Practice, Oxford University Press: New York, 1998.

[2] Welton, T., Room-Temperature Ionic Liquids. Solvents for Synthesis and Catalysis. Chem. Rev. 1999, 99, 2071-2084.

[3] Dupont, J.; De Souza, R. F.; Suarez, P. A. Z., Ionic Liquid (Molten Salt) Phase Organometallic Catalysis. Chem. Rev. 2002, 102, 3667-3692.

[4] Hernandez, O. R., ToTreat or Not to Treat? Applying Chemical Engineering Tools and a Life Cycle Approach to Assessing the Level of Sustainability of a Clean-up Technology. Green Chem. 2004, 6, 395-400.

[5] Clift, R., Sustainable Development and Its Implications for Chemical Engineering. Chem. Eng. Sci. 2006, 61, 4179-4187.

[6] Fischer, T.; Sethi, A.; Welton, T.; Woolf, J., Diels-Alder Reactions in Room-temperature Ionic Liquids. Tetrahedron Lett. 1999, 40, 793-796. 
[7] Boon, J. A.; Levisky, J. A.; L., P. J.; Wilkes, J. S., Friedel-Crafts Reactions in AmbientTemperature Molten Salts. J. Org. Chem. 1986, 51, 480-483.

[8] Brown, R. A.; Pollet, P.; McKoon, E.; Eckert, C. A.; Liotta, C. L.; Jessop, P. G., Asymmetric Hydrogenation and Catalyst Recycling Using Ionic Liquid and Supercritical Carbon Dioxide. J. Am. Chem. Soc. 2001, 123, 1254-1255.

[9] Chauvin, Y.; Mussmann, L.; Olivier, H., A Novel Class of Versatile Solvents for TwoPhase Catalysis: Hydrogenation, Isomerization, and Hydroformylation of Alkenes Catalyzed by Rhodium Complexes in Liquid 1,3-Dialkylimidazolium Salts. Angew. Chem., Int. Ed. Engl. 1996, 34, 2698-2700.

[10] Favre, F.; Olivier-Bourbigou, H.; Commereuc, D.; Saussine, L., Hydroformylation of 1-Hexene with Rhodium in Non-aqueous Ionic Liquids : How to Design the Solvent and the Ligand to the Reaction. Chem. Commun. 2001, 1360-1361.

[11] Dullius, J. E. L.; Suarez, P. A. Z.; Einloft, S.; de Souza, R. F.; Dupont, J., Selective Catalytic Hydrodimerization of 1,3-Butadiene by Palladium Compounds Dissolved in Ionic Liquids. Organometallics 1998, 17 (5), 815-819.

[12] Kaufmann, D. E.; Nouroozian, M.; Henze, H., Molten Salts as an Efficient Medium for Palladium Catalyzed C-C Coupling Reactions. Synlett. 1996, 1091-1092.

[13] Matthews, C. J.; Smith, P. J.; Welton, T., Palladium Catalysed Suzuki Cross-coupling Reactions in Ambient Temperature Ionic Liquids. Chem. Commun. 2000, 1249-1250.

[14] Parvulescu, V. I.; Hardacre, C., Catalysis in Ionic Liquids. Chem. Rev. 2007, 107, 2615-2665.

[15] Hallett, J. P.; Welton, T., Room-Temperature Ionic Liquids: Solvents for Synthesis and Catalysis. 2. Chem. Rev. 2011, 111, 3508-3576.

[16] Olivier-Bourbigou, H.; Magna, L.; Morvan, D., Ionic Liquids and Catalysis: Recent Progress from Knowledge to Applications. Appl. Catal. A 2010, 373, 1-56.

[17] Lee, J. W.; Shin, J. Y.; Chun, Y. S.; Jang, H. B.; Song, C. E.; Lee, S., Toward Understanding the Origin of Positive Effects of Ionic Liquids on Catalysis: Formation of More Reactive Catalysts and Stabilization of Reactive Intermediates and Transition States in Ionic Liquids. Acc. Chem. Res. 2010, 43 (7), 985-994.

[18] Stark, A., Ionic Liquid Structure-Induced Effects on Organic Reactions. Top Curr. Chem. 2009, 290, 41-81.

[19] Wasserscheid, P.; Keim, W., Ionic Liquids-New "solution" for Transition Metal Catalysis. Angew Chem, Int. Ed. 2000, 39, 3772-3789.

[20] Sheldon, R., Catalytic Reactions in Ionic Liquids. Chem. Commun. 2001, 2399-2407.

[21] Gharnati, L.; Doering, M.; Arnold, U., Catalytic Oxidation with Hydrogen Peroxide in Ionic Liquids. Curr. Org. Synth. 2009, 6 (4), 342-361. 
[22] Betz, D.; Altmann, P.; Cokoja, M.; Herrmann, W. A.; Kuehn, F. E., Recent Advances in Oxidation Catalysis Using Ionic Liquids as Solvents. Coord. Chem. Rev. 2011, 255 (13-14), 1518-1540.

[23] Muzart, J., Ionic Liquids as Solvents for Catalyzed Oxidations of Organic Compounds. Adv. Synth. Catal. 2006, 348, 275-295.

[24] Walling, C., Intermediates in the Reactions of Fenton Type Reagents. Acc. Chem. Res. 1998, 31, 155-157.

[25] Hu, X. K.; Zhu, L. F.; Guo, B.; Liu, Q. Y.; Li, G. Y.; Hu, C. W., Hydroxylation of Benzene to Phenol via Hydrogen Peroxide in Hydrophilic Triethylammonium Acetate Ionic Liquid. Chem. Res. Chin. Univ. 2011, 27 (3), 503-507.

[26] Zhang, J.; Tang, Y.; Li, G. Y.; Hu, C. W., Room Temperature Direct Oxidation of Benzene to Phenol Using Hydrogen Peroxide in the Presence of Vanadium-substituted Heteropolymolybdates. Appl. Catal. A 2005, 278, 251-261.

[27] Zhong, Y. K.; Li, G. Y.; Zhu, L. F.; Yan, Y.; Wu, G.; Hu, C. W., Low Temperature Hydroxylation of Benzene to Phenol by Hydrogen Peroxide over Fe/activated Carbon Catalyst. J. Mol. Catal. A 2007, 272, 169-173.

[28] Jian, M.; Zhu, L. F.; Wang, J. Y.; Zhang, J.; Li, G. Y.; Hu, C. W., Sodium Metavanadate Catalyzed Direct Hydroxylation of Benzene to Phenol with Hydrogen Peroxide in Acetonitrile Medium. J. Mol. Catal. A 2006, 253, 1-7.

[29] Bianchi, D.; Bertoli, M.; Tassinari, R.; Ricci, M.; Vignola, R., Ligand Effect on the Ironcatalysed Biphasic Oxidation of Aromatic Hydrocarbons by Hydrogen Peroxide J. Mol. Catal. A 2003, 204, 419-424.

[30] Bianchi, D.; Balducci, L.; Bortolo, R.; D' Aloisio, R.; Ricci, M.; Span, G.; Tassinari, R.; Tonini, C.; Ungarellia, R., Oxidation of Benzene to Phenol with Hydrogen Peroxide Catalyzed by a Modified Titanium Silicalite (TS-1B). Adv. Synth. Catal. 2007, 349, 979-986.

[31] Peng, J. J.; Shi, F.; Gu, Y. L.; Deng, Y. Q., Highly Selective and Green Aqueous-Ionic Liquid Biphasic Hydroxylation of Benzene to Phenol with Hydrogen Peroxide. Green Chem. 2003, 5 (2), 224-226.

[32] Hu, X. K.; Zhu, L. F.; Wang, X. Q.; Guo, B.; Xu, J. Q.; Li, G. Y.; Hu, C. W., Active Species Formed in a Fenton-Like System in the Medium of Triethylammonium Acetate Ionic Liquid for Hydroxylation of Benzene to Phenol. J. Mol. Catal. A 2011, 342-343, 41-49.

[33] Hu, X. K., Study on Hydroxylation of Benzene in Triethylammonium Acetate Ionic Liquid. Chinese Doctoral Dissertation 2011.

[34] Ley, S. V.; Madin, A., in: Trost, B. M.; Flemming, I. (Eds.), Comprehensive Organic Synthesis, Vol. 7, 305-327, Pergamon Press, Oxford, 1991. 
[35] Hudlick, M., Oxidations in Organic Chemistry, American Chemical Society: Washington, DC, 1990.

[36] Sheldon, R. A.; Kochi, J. K., Metal Catalyzed Oxidationd of Organic Compounds, Academic Press, New York, 1984.

[37] Bobbitt, J. M.; Br ${ }^{\circ}$ uckner, C., Organic Reactions, John-Wiley \& Sons, New York, 2009.

[38] Anelli, P. L.; Biffi, C.; Montanari, F.; Quici, S., J. Org. Chem. 1987, 52, 2559-2562.

[39] Fall, A.; Sene, M.; Gaye, M.; Gomez, G.; Fall, Y., Ionic Liquid-Supported TEMPO as Catalyst in the Oxidation of Alcohols to Aldehydes and Ketones. Tetrahedron Lett. 2010, 51 (34), 4501-4504.

[40] Hoover, J. M.; Stahl, S. S., Highly Practical Copper(I)/TEMPO Catalyst System for Chemoselective Aerobic Oxidation of Primary Alcohols. J. Am. Chem. Soc. 2011, 133 (42), 16901-16910.

[41] Ma, S. M.; Liu, J. X.; Li, S. H.; Chen, B.; Cheng, J. J.; Kuang, J. Q.; Liu, Y.; Wan, B. Q.; Wang, Y. L.; Ye, J. T.; Yu, Q.; Yuan, W. M.; Yu, S. C., Development of a General and Practical Iron Nitrate/TEMPO-Catalyzed Aerobic Oxidation of Alcohols to Aldehydes/Ketones: Catalysis with Table Salt. Adv. Synth. Catal. 2011, 353 (6), 1005-1017.

[42] Hoover, J. M.; Steves, J. E.; Stahl, S. S., Copper(I)/TEMPO-catalyzed aerobic oxidation of primary alcohols to aldehydes with ambient air. Nature Protoc. 2012, 7 (6), 1161-1166.

[43] Gheorghe, A.; Chinnusamy, T.; Cuevas-Yanez, E.; Hilgers, P.; Reiser, O., Combination of Perfluoroalkyl and Triazole Moieties: A New Recovery Strategy for TEMPO. Org. Lett. 2008, 10 (19), 4171-4174.

[44] Liu, R.; Liang, X.; Dong, C.; Hu, X., Transition-Metal-Free: A Highly Efficient Catalytic Aerobic Alcohol Oxidation Process. J. Am. Chem. Soc. 2004, 126, 4112-4113.

[45] Wang, S. S.; Popovic, Z.; Wu, H. H.; Liu, Y., A Homogeneous Mixture Composed of Vanadate, Acid, and TEMPO Functionalized Ionic Liquids for Alcohol Oxidation by H2O2. ChemCatChem2011, 3 (7), 1208-1213.

[46] Jiang, N.; Ragauskas, A. J., TEMPO-Catalyzed Oxidation of Benzylic Alcohols to Aldehydes with the H2O2/HBr/Ionic Liquid [bmim] PF6 System. Tetrahedron Lett. 2005, 46 (19), 3323-3326.

[47] Karimi, B.; Badreh, E., SBA-15-Functionalized TEMPO Confined Ionic Liquid: an Efficient Catalyst System for Transition-Metal-Free Aerobic Oxidation of Alcohols with Improved Selectivity. Org. Biomol. Chem. 2011, 9 (11), 4194-4198.

[48] Chhikara, B. S.; Chandra, R.; Tandon, V., Oxidation of Alcohols with Hydrogen Peroxide Catalyzed by a New Imidazolium Ion Based Phosphotungstate Complex in Ionic Liquid. J. Catal. 2005, 230 (2), 436-439.

[49] Bianchini, G.; Crucianelli, M.; De Angelis, F.; Neri, V.; Saladino, R., Highly Efficient $\mathrm{C}-\mathrm{H}$ Insertion Reactions of Hydrogen Peroxide Catalyzed by Homogeneous and Het- 
erogeneous Methyltrioxorhenium Systems in Ionic Liquids. Tetrahedron Lett. 2005, 46 (14), 2427-2432.

[50] Chen, L.; Zhou, T.; Chen, L.; Ye, Y.; Qi, Z.; Freund, H.; Sundmacher, K., Selective Oxidation of Cyclohexanol to Cyclohexanone in the Ionic Liquid 1-Octyl-3-Methylimidazolium Chloride. Chem. Commun. 2011, 47 (33), 9354-9356.

[51] Usui, Y.; Sato, K., A Green Method of Adipic Acid Synthesis: Organic Solvent- and Halide-Free Oxidation of Cycloalkanones with 30\% Hydrogen Peroxide. Green Chem. 2003, 5, 373-375.

[52] Singh, P. P.; Ambika; Chauhan, S. M. S., Chemoselective Epoxidation of Electron Rich and Electron Deficient Olefins Catalyzed by Meso-Tetraarylporphyrin Iron(III) Chlorides in Imidazolium Ionic Liquids. New J. Chem. 2012, 36 (3), 650-655.

[53] Liu, G.; Hou, M. Q.; Song, J. Y.; Zhang, Z. F.; Wu, T. B.; Han, B. X., Ni2+-Containing Ionic Liquid Immobilized on Silica: Effective Catalyst for Styrene Oxidation with H2O2 at Solvent-Free Condition. J. Mol. Catal. A 2010, 316 (1-2), 90-94.

[54] Yamaguchi, K.; Yoshida, C.; Uchida, S.; Mizuno, N., Peroxotungstate immobilized on ionic liquid-modified silica as a heterogeneous epoxidation catalyst with hydrogen peroxide. J. Am. Chem. Soc. 2005, 127 (2), 530-531.

[55] Berardi, S.; Bonchio, M.; Carraro, M.; Conte, V.; Sartorel, A.; Scorrano, G., Fast Catalytic Epoxidation with $\mathrm{H} 2 \mathrm{O} 2$ and [gamma-SiW10O36(PhPO)2]4 in Ionic Liquids under Microwave Irradiation. J. Org. Chem. 2007, 72 (23), 8954-8957.

[56] Liu, L. L.; Chen, C. C.; Hu, X. F.; Mohamood, T.; Ma, W. H.; Lin, J.; Zhao, J. C., A Role of Ionic Liquid as an Activator for Efficient Olefin Epoxidation Catalyzed by Polyoxometalate. New J. Chem. 2008, 32 (2), 283-289. 